\title{
Bipolar Scales with Pictorial Anchors: Some Characteristics and a Method for their Use
}

\author{
Arthur D. Beard \\ Central Michigan University
}

\begin{abstract}
Visual aesthetic preferences seem to be based upon a judgmental mechanism that processes nonverbal cues. Yet the usual methods for measuring these cues are verbal. A nonverbal method for identifying and measuring the component cues present in slides of nonrepresentational paintings is described. The method was used to develop pictorially anchored scales that were easy for subjects to use and that elicited reliable cue ratings.
\end{abstract}

What elements in a painting are related to the amount of time a person spends looking at it? What styling components in a new automobile are related to its sales? What aspects of a person's appearance are related to his or her social success? Or, more generally, what variables in a nonverbal, visually perceived aesthetic stimulus predict a person's global reaction to it? Although the answers to this type of question, which may be of significance in the achievement of both personal and commercial goals, have been of great interest to scientists for over a century (e.g., Fechner, 1865), they have been difficult to obtain. Berlyne (1971), for example, described experimental aesthetics as being in a state of "protracted infancy."

One factor that may be inhibiting progress is the manner of taking measurements. As Nun-

APPLIED PSYCHOLOGICAL MEASUREMENT

Vol. 3, No. 4 Fall 1979 pp. 469-480

(C) Copyright 1979 West Publishing Co. nally, Lemond, and Wilson (1977) noted, the only way to establish values for most of the important variables in a nonverbal, visually perceived aesthetic stimulus is in terms of subjects' reactions. These reactions are typically measured through the use of verbally anchored rating scales (Berlyne, 1974). The question is, Is it reasonable to use verbally anchored rating scales to establish values for variables that are elements of a nonverbal stimulus?

\section{The Nature of the Visual Aesthetic Process}

If the global reaction of interest (e.g., voluntary visual attention) is viewed as the output of a process whose inputs are the variables to be measured, there must be an inquiry into the nature of the process. Is the process one that usually inputs and processes verbal material, even though the stimulus itself is without verbal elements? Indeed, this would be the case if a viewer of a painting translated his/her visual impressions into verbalizable thoughts as his/her eyes roamed over the canvas. But does this describe the nature of the visual aesthetic experience and is the word-encoded thought a typical characteristic?

In a paper exploring critics' evaluations of plays, Einhorn and Koelb (1974, p. 7) stated, "Whether the final categorization is a diagnosis 
of a disease or a judgment that a work of art is 'great,' the same mental processes appear to be at work." This suggests that the process generating a global reaction to a stimulus is the same in its fundamental character regardless of the nature of the stimulus or of the situation in which the stimulus is perceived. But writers in the area of visual aesthetics seem almost unanimous in disagreeing with that idea (e.g., Chandler, 1934; Henze, 1957; Munro, 1970). They feel that an important characteristic of the process associated with the aesthetic perception of nonverbal, visually perceived stimuli is its nonverbal nature, which is not characteristic of typical analytical thought processes. For example, Alexander (1960) had subjects judge the similarity of the eight simple forms which are shown in Figure 1. The subjects' efforts to verbalize the basis for their judgments showed little relation to the dimensions uncovered by statistical techniques and varied from one subject to another. Alexander concluded that "we do not have words for what we do with our eyes" (p. 369). Related experimental findings and opinions are also found in Bannister and Mair (1968), Child (1969), Mirels and Efland (1970), and Wright and Rainwater (1962).

Given the above, it may be proposed that although untrained persons may not be able to supply appropriate verbal anchors for rating scales, experts skilled in the vocabulary of aesthetic experience could ably supply the anchors for laymen to use. However, there is some question about even the experts' ability to supply useful verbal anchors. Gibson (1966, p. 240) observed, "No one, artist or psychologist, has ever been quite sure what a line was, or a boundary, margin, contour, texture, pattern or form."

It seems, therefore, that the aesthetic experience regarding nonverbal visual stimuli does not involve the mediation of words. And, indeed, medical science has shown that one side of the human brain specializes in processing nonverbal information (Ornstein, 1975).

\section{A Problem in Using Verbally Anchored Scales?}

The apparent nonverbal nature of the processing of visual aesthetic stimuli, and of the stimuli themselves, may make it difficult for a verbal methodology to establish adequate numerical representations of the variables actually present in the stimuli. It may also limit the investigator's ability to establish construct validity for the variables. Construct validity is usually thought of in respect to some aspect of a person or his/her experience, not to objects such as paintings. But since mechanical and optical devices cannot be used to measure important dimensions such as "movement" or "depth" in a painting, a person must be used as the sensing and measuring instrument. Most variables in the object are not present in any meaningful sense without human processing. Therefore, the constructs of interest to the experimental aesthetician may be considered as traits belonging to both the person and to the stimulus in combination.

\section{Figure 1}

Eight Stimuli Used by Alexander (1960)

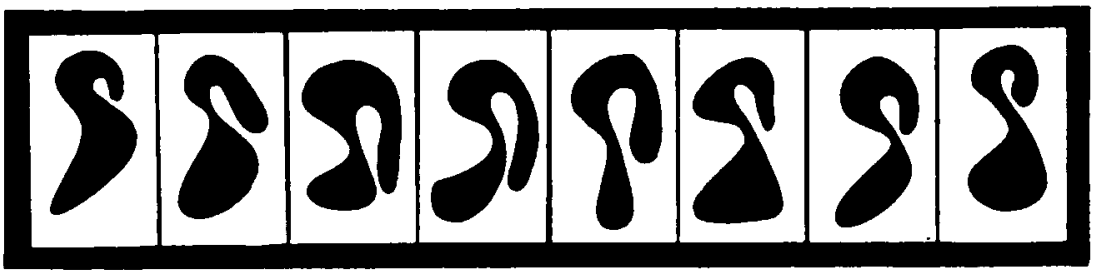


According to Nunnally (1967), one important aspect of construct validity is the name of the construct: ". . . the name is 'valid' only to the extent that it accurately communicates to other scientists the kind of observables being studied" (p. 95). In experimental aesthetics, the name of a variable is often a hyphenated pair of bipolar anchors lifted from the scale used to measure the variable, e.g., curved-angular. To the extent that the nonverbal nature of the process inhibits the establishment of denotative meaning for the words forming the anchors (and consequently for the construct's name), the fidelity of communication achieved by the name, and therefore its construct validity, will be inhibited.

\section{A Solution?}

If the fidelity of communication is reduced by the process of encoding a nonverbal, visually perceived object into words, why not eliminate the encoding step? This suggests substituting nonverbal visual referents, pictures, for the usual verbal anchors on rating scales. Of course, to avoid substituting vague pictorial abstractions for vague verbal abstractions, the pictorial anchors should be fully detailed and in full color.

The feasibility of using pictorial anchors is doubtful, however, for the following reasons: (1) the pictures on a rating form would be far too small to show the necessary detail unless the rating form were tablecloth-size; (2) reproducing full-color pictures for use as scale anchors would be prohibitively expensive; (3) persons likely to be subjects are accustomed to using verbally anchored scales and would find pictorially anchored scales too difficult to use; and (4) measures obtained with such scales would be unreliable.

\section{Why Bother with Pictorially Anchored Scales?}

The value of efforts to develop pictorially anchored scales in spite of the doubts lies in at least four areas. First, as noted above, the pic- torial anchors may enhance the measured construct's validity. Second, if the stimuli being judged are nonverbal, visually perceived, and aesthetic, then pictorially anchored scales may uniquely identify the appropriate variables for inclusion in models of aesthetic judgment. Third, the use of pictorial anchors may allow use of the scales by subjects who are illiterate or who cannot interpret the verbal anchors, for instance, younger children. And fourth, the picture as a visual referent will eliminate problems in translating verbal anchors from one language to another, therefore facilitating some types of crosscultural research.

\section{A Method for Using Pictorially Anchored Scales}

\section{Displaying the Anchors}

The solution to the problems of size and expense lies in separating the form used to record subjects' ratings from the pictorial anchors. The anchors can be displayed as large full-color prints or as projections of full-color slides. If the prints or projections are large enough, many subjects can use the anchors simultaneously. A large drawing of a scale can be displayed between the pictures so that the pictures' function as scale anchors is obvious. After a subject makes a rating using one of the large scales, he/she can record the rating on a form similar to the one shown in Figure 2.

The anchors on the scales in the rating form are simply thumbnail sketches of the large fullcolor reproductions and, as such, are merely reference points. A subject can use them to identify the correct scale, as well as to locate the correct point on that scale for recording the rating already made in respect to the large display.

\section{Identifying the Anchors to be Displayed}

An appropriate method for identifying pictorial anchors should minimize the subjects' use of words. One such method is nonmetric multidimensional scaling (MDS), which requires only 
Figure 2

Form for Recording Ratings Made with Pictorially Anchored Scales

Date

Painting

Subject

I
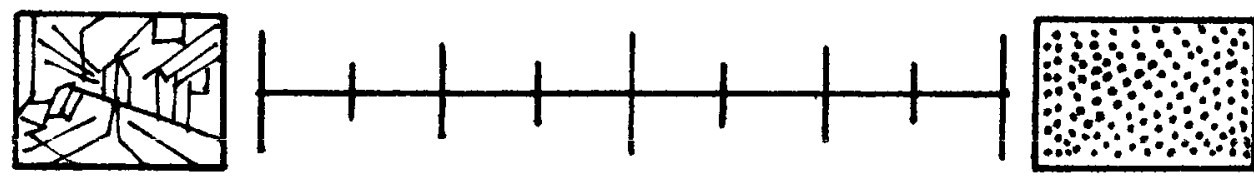

II
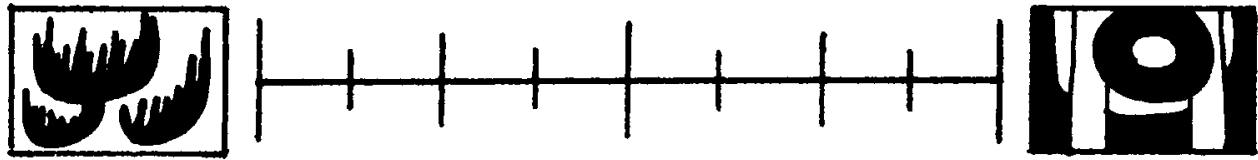

III
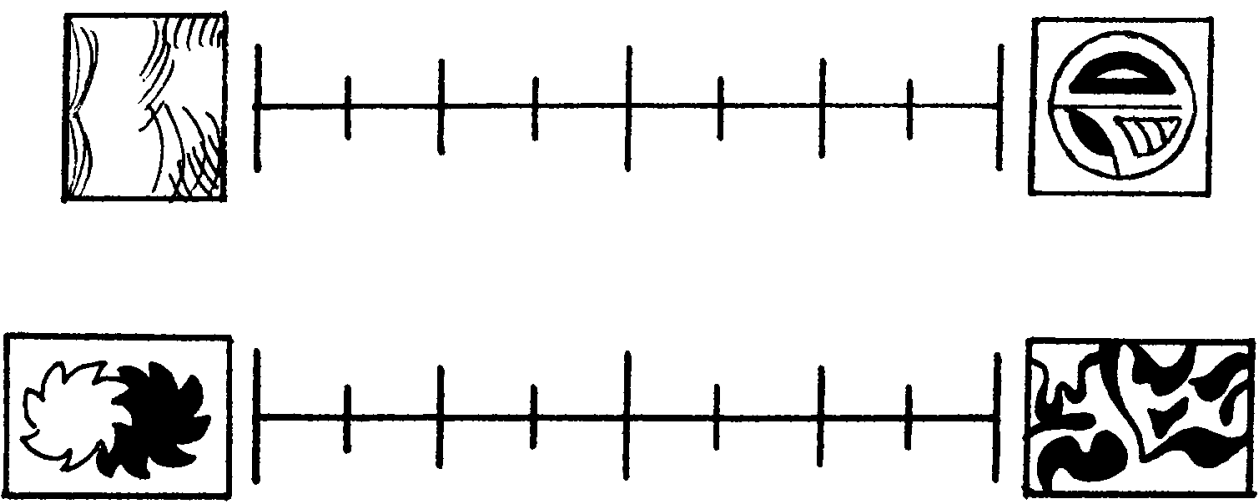

$\mathrm{V}$
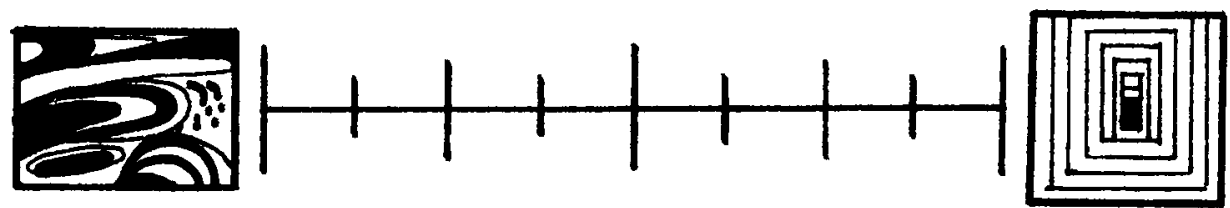
that subjects provide ratings or rankings of the similarity or dissimilarity of members of stimulus pairs. The MDS output represents an orthogonally dimensioned space in which the stimuli are positioned with respect to each other. The two stimuli farthest away from each other on a straight line passing through the center of the MDS-generated similarity-dissimilarity space can be interpreted as representing the extreme values of a perceptual dimension. There is some justification, therefore, in using those stimuli as anchors for a scale designed to measure the dimension whose extremes they represent.

\section{The Type of Stimulus and the Aesthetic Experience}

The selection of stimuli is critical in a study purporting to measure aspects of the aesthetic experience. The rationale for constructing and testing pictorially anchored scales is the apparent nonverbal nature of this type of experience. The stimuli, then, must be ones that are likely to be associated with such an experience. Various scholars (e.g., Arnheim, 1966; Berleant, 1964; Moles, 1966; Nidorf \& Argabrite, 1970; Zenzen, 1976) have suggested that any stimulus may evoke the process of aesthetic perception and reaction under some conditions. The combined views of Ashmore (1955), Child (1969), and Lindauer (1971) suggest that the more representational a work of art is (i.e., the more it is photographic in appearance), the more likely it is to invoke a verbal-numeric-utilitarian brain process that is not characteristic of the aesthetic experience. The works of art that seem to be the type most likely to invoke the aesthetic experience are nonrepresentational and especially nonobjective (not meant to represent, nor to be an abstraction of, an object).

\section{Objectives of the Research}

The research described below was undertaken to explore the use of pictorially anchored rating scales to measure variables present in nonverbal, visually perceived aesthetic stimuli. The objectives were (1) to see if a specific technique for displaying the anchors would work; (2) to see how difficult the pictorially anchored scales would be to use; and (3) to measure the test-retest reliability of the scales.

\section{Method}

\section{Subjects}

The data and results to be reported are from eight separate studies, each of which focused on the aesthetic judgments of one subject. All the subjects would be considered above average in language skills and in their interest in aesthetic matters. The findings of these studies were not intended to be generalized to other persons and should be viewed as the results of an exploratory first step.

\section{Stimuli}

All the stimuli used were $35 \mathrm{~mm}$ color slides of nonrepresentational paintings selected by an expert in art history and aesthetics. The stimuli were organized into two sets: One was composed of 15 slides; the other, of 100 slides. The sets were mutually exclusive, but both sets were carefully selected to represent the broad range of executions in the nonrepresentational category. Therefore, within subjects, there can be confidence in generalizing to the universe of nonrepresentational paintings to the extent that the slides typify that universe.

\section{Identifying the Perceptual Dimensions}

The slides in the set of 15 were projected in pairs onto a white wall in the investigator's office, separately to each subject. Similarity-dissimilarity judgments of the members of every possible pair were provided by each subject on an 11-point scale. The slides were presented in the same order to every subject. The subject's only task at this point was to rate the similarity 
of the two slides in the pair. No verbalizations were requested or recorded. The subjects controlled the amount of time that they spent examining each pair.

The similarity-dissimilarity judgments for each subject were submitted separately to Kruskal's (1964a, 1964b) nonmetric multidimensional scaling procedure. The smallest number of dimensions consistent with Kruskal's criterion for "good" fit (stress $\leqslant .05$ ) and with the position of the "elbow" in a plot of the number of dimensions vis-à-vis the stress statistic was determined. The locations of the 15 slides on each dimension were plotted. The slides were arranged in the plotted positions separately for each subject, two dimensions at a time on a light table. This was done to see if a rotation of the dimensions would be necessary in order to identify the slides at the extremes of the perceptual dimensions each subject used. The slides at the extremes of the unrotated dimensions were judged appropriate for use.

The number (see Table 3) and interpretation of the uncovered dimensions varied dramatically from subject to subject. This variation raises questions about the appropriateness of averaging similarity-dissimilarity ratings or of processing several subjects in one run of an individual differences multidimensional scaling procedure such as INDSCAL (Carroll \& Chang, 1970). These issues are outside the scope of this report, but the observed variation supports the idiosyncratic approach to scale development employed here.

\section{The Pictorially Anchored Rating Scales}

Separately for each subject, large full-color printed reproductions of the paintings in the slides at the extremes of each dimension were used to label the end points of rating scales on a large ( 3 foot $\times 4$ foot) display board. The display board was painted flat black in order to enhance the visibility of the reproductions. The scales were laid out with $1 / 4$ inch white striping tape between the two pictorial anchors. The board and reproductions were constructed and mounted so that modifications could be made rapidly to represent the different perceptual structures of the eight subjects. Each scale was uniquely identified by a roman numeral and by its pictorial anchors. The general appearance of the display board is that of the rating form shown in Figure 2. Printed forms for recording the ratings were prepared to approximate the appearance of the display board. Note that the subjects performed the rating task while looking at the large fullcolor printed reproductions on the display board. The rating forms were used only to record a rating once it had been made. For each subject, then, there was a tailor-made set of large 8-point rating scales on the display board and a set of forms for recording the ratings.

\section{Tasks}

Each subject evaluated the randomly ordered set of 100 slides using the pictorially anchored scales. Each slide was rated on all the scales before the next slide was viewed. In order to measure test-retest reliability, 50 randomly selected slides were evaluated a second time after the passage of at least 1 week and a maximum of 3 weeks. The order of the slides was the same for all subjects.

After each rating session, the subject used a 6point scale to rate the difficulty of using each individual scale. The time each subject required to use each set of scales was also recorded.

\section{Analysis}

The analysis was conducted in three main parts:

1. The subjects' difficulty ratings of each scale and the times required to use the set of scales were averaged separately and used as two measures of difficulty.

2. Pearson product-moment correlations between ratings on the $\mathbf{5 0}$ repeated stimuli in 
the first and second sessions were used to measure test-retest reliability.

3. Separately for each subject, intercorrelations of the measures obtained with his/her pictorially anchored scales were calculated to gauge the orthogonality of the perceptual dimensions in the 100-slide stimulus set.

In addition, the relationships among the outputs of the three parts of the analysis were ex- amined in various ways and are described where appropriate below.

\section{Results}

\section{Difficulty}

Table 1 shows that, on the average, the pictorially anchored scales were rated in the easy half of the 6-point easy-difficult scale and there-

Table 1

Subjective Difficulty Ratings by Scale within Subject, and by Rating Session

\begin{tabular}{|c|c|c|c|c|c|c|c|c|c|}
\hline \multirow{2}{*}{$\begin{array}{c}\text { Pictorially } \\
\text { Anchored } \\
\text { Scale } \\
\end{array}$} & \multicolumn{8}{|c|}{ Subject } & \multirow{2}{*}{$\begin{array}{c}\text { Mean } \\
\text { Difficulty } \\
\text { Ratings }\end{array}$} \\
\hline & 1 & 2 & 3 & 4 & 5 & 6 & 7 & 8 & \\
\hline $\mathrm{Pl}$ & $1,2^{a}$ & 1,1 & 4,2 & 4,4 & 2,1 & 1,1 & 2,1 & 3,3 & $\mathrm{~b}$ \\
\hline $\mathrm{P} 2$ & 4,5 & 5,2 & 2,3 & 6,6 & 2,1 & 2,2 & 5,4 & 3,2 & \\
\hline P3 & 1,1 & 2,1 & 3,2 & 5,5 & & 2,2 & 1,2 & 4,3 & \\
\hline P4 & 2,2 & 5,2 & 4,4 & 3,4 & & & 3,3 & 4,3 & \\
\hline P5 & 3,5 & & 6,4 & & & & 3,1 & 4,3 & \\
\hline \multicolumn{10}{|l|}{ Means: } \\
\hline lst Session & 2.2 & 3.3 & 3.8 & 4.5 & 2.0 & 1.7 & 2.8 & 3.6 & 3.1 \\
\hline 2nd Session & 3.0 & 1.5 & 3.0 & 4.8 & 1.0 & 1.7 & 2.2 & 2.8 & 2.6 \\
\hline Change & .8 & $(1.8)$ & $(.8)$ & .3 & $(1.0)$ & 0 & $(.6)$ & $(.8)$ & $(.5)$ \\
\hline Both & & & & & & & & & \\
\hline $\begin{array}{l}\text { Sessions C } \\
\text { S.D. }\end{array}$ & 2.5 & 2.7 & 3.5 & 4.6 & 1.7 & 1.7 & 2.6 & 3.3 & 2.9 \\
\hline lst Session & 1.2 & 1.8 & 1.3 & 1.1 & 0 & .5 & 1.3 & .5 & \\
\hline 2nd Session & 1.7 & .5 & .9 & .8 & 0 & .5 & 1.2 & .4 & \\
\hline Change & .5 & $(1.3)$ & $(.4)$ & $(.3)$ & 0 & 0 & $(.1)$ & $(.1)$ & \\
\hline
\end{tabular}

$a_{\text {Read: }}$ Subject 1 rated the difficulty of scale $P 1$ as 1 after the first rating session, and as 2 after the second rating session. $l=$ Extremely easy to use; 6 = Extremely difficult to use.

$\mathrm{b}_{\text {Scale }} \mathrm{PI}$ for subject $\mathrm{l}$ is different from scale Pl for other subjects; likewise for other subjects and other scales. Therefore, means across subjects for individual scales are meaningless and were not calculated.

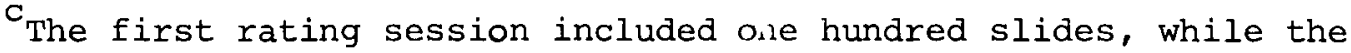
second rating session included fifty. Therefore the first session's figures were given twice the weight of the second's when the means were calculated. 
fore may be described as easy to use. There was considerable variation across subjects, as well as from scale to scale within subjects. One subject, Subject 4, found all her scales difficult to use in the second rating session and all but one difficult to use in the first session. Although there was an apparent overall drop in perceived difficulty from the first to the second rating session, a $t$-test for related measures indicated that the change was not great enough to be statistically significant at the .05 level. The standard deviations in Table 1 show that there was a tendency for the difficulty ratings to become more uniform from the first to the second rating sessions. This seems largely due to several scales being rated as less difficult after the second session.

Table 2 shows the average number of seconds required by each subject to use each of his/her pictorially anchored scales. The time required to use a specific individual scale was not recorded. Therefore, an entry in the columns for the first and second rating sessions was obtained by dividing the total time a subject needed to rate all the session's slides by the product of the number of slides rated and the number of scales used per slide. Table 2 shows the wide differences across subjects and the drop in value from the first to the second rating session that were apparent in Table 1. A $t$-test for related measures showed that the change in time required was statistically significant beyond the .01 level $(t=4.088, d f=7)$.

The Pearson product-moment correlation between mean subjectively rated difficulty and mean time required was -.60 but was not statistically significant at the .05 level.

\section{Reliability}

Table 3 displays the test-retest reliabilities separately for each subject and for each pictorially anchored scale. The mean of the individual subjects' reliabilities was .58 . Generally, the pictorially anchored scales seemed to gen-

Table 2

Mean Time (in Seconds) Required to Use Pictorially Anchored Rating Scales

\begin{tabular}{ccccc}
\hline & \multicolumn{4}{c}{ Rating Session } \\
\cline { 2 - 5 } Subject & First & Second & Change & Both \\
\hline 1 & 7.8 & 6.7 & $(.9)$ & $7.4^{\mathrm{a}}$ \\
2 & 5.3 & 3.0 & $(2.3)$ & 4.5 \\
3 & 5.8 & 3.6 & $(2.2)$ & 5.1 \\
4 & 7.2 & 3.3 & $(3.9)$ & 5.9 \\
5 & 7.8 & 6.6 & $(1.2)$ & 7.4 \\
6 & 15.4 & 9.2 & $(6.2)$ & 13.3 \\
7 & 7.3 & 4.3 & $(3.0)$ & 6.3 \\
8 & 4.0 & 3.1 & $(.9)$ & 3.7 \\
Mean & 7.6 & 5.0 & $(2.6)$ & 6.7 \\
S.D. & 3.2 & 2.1 & $(1.1)$ & \\
\hline ahe first rating session included one hundred \\
Thides, while the second session included \\
slides \\
fifty. Therefore the first session's figures \\
were given twice the weight of the second \\
session's figures when the means for both \\
sessions were calculated.
\end{tabular}




\begin{tabular}{|c|c|c|c|c|c|c|c|c|}
\hline \multirow{2}{*}{$\begin{array}{c}\text { Pictorially- } \\
\text { Anchored } \\
\text { Scale }\end{array}$} & \multicolumn{8}{|c|}{ subject } \\
\hline & 1 & 2 & 3 & 4 & 5 & 6 & 7 & 8 \\
\hline $\mathrm{Pl}$ & .44 & .65 & .48 & .59 & .56 & .85 & .66 & .55 \\
\hline $\mathrm{P} 2$ & .33 & .61 & .28 & .59 & .60 & .60 & .70 & .43 \\
\hline P3 & .04 & .66 & .37 & .50 & & .77 & .62 & .69 \\
\hline$P 4$ & .39 & .70 & .44 & .64 & & & .63 & .53 \\
\hline $\mathrm{P} 5$ & .15 & & .57 & & & & .92 & .66 \\
\hline Subject Means & .28 & .66 & .43 & .58 & .58 & .76 & .74 & .58 \\
\hline
\end{tabular}

erate moderately reliable measures, although two of Subject 1's obviously did not and those of some of the other subjects would be considered highly reliable (e.g., Scale P5 of Subject 7). The Pearson product-moment correlation between the reliability and difficulty ratings of the 33 scales was -.06 and was not statistically significant; nor did a scatterplot reveal a visually perceptible hint of a curvilinear relationship.

\section{Intercorrelations of the Measures}

Separately for each subject, Pearson productmoment correlation coefficients were calculated for each possible pair of scales. The intercorrelation matrices are shown in Table 4.

Table 4 shows that the apparent degree of orthogonality of the MDS-generated perceptual dimensions in the 100 -slide stimulus set varied from subject to subject, but in general the ratings were not independent.

It may be asked if the intercorrelations are related to difficulty rather than to an accurate representation of the dimensional relationships in the stimulus set. For example, if a subject experienced difficulty in using some, but not all, of his/her scales, the subject may have marked the scales that were difficult to use similarly to those that were easy to use, thereby accounting for the intercorrelations. To explore this possibility, the intercorrelations for each subject were summarized in two ways: (1) the mean intercorrela- tion and (2) the proportion of pairs of scales with significant correlations. The relationships of these summary measures with the mean difficulties for both rating sessions, shown in Table 1 , and the mean time required for both rating sessions, shown in Table 2, were evaluated with Spearman's rank order correlation coefficient: None of the four relationships was significant at the .05 level.

\section{Discussion}

\section{Ease of Use and Reliability}

On the average, the subjects in this study found the pictorially anchored scales easy to use. This is encouraging, since easy-to-use scales should be usable by a wide range of subjects. However, such encouragement must be tempered by the fact that the subjects were probably above average in their interest in paintings. The difficulty ratings and times required to use the scales suggest (unsurprisingly) that the ease of use increases with practice, although support for this was statistically significant only for the time data.

The results show that generally reliable measures of component dimensions in nonverbal visually perceived aesthetic stimuli can be obtained using detailed full-color pictures as scale anchors. The reliability of the measures did not seem to be linearly or curvilinearly related to their ease of use. Further work is re- 


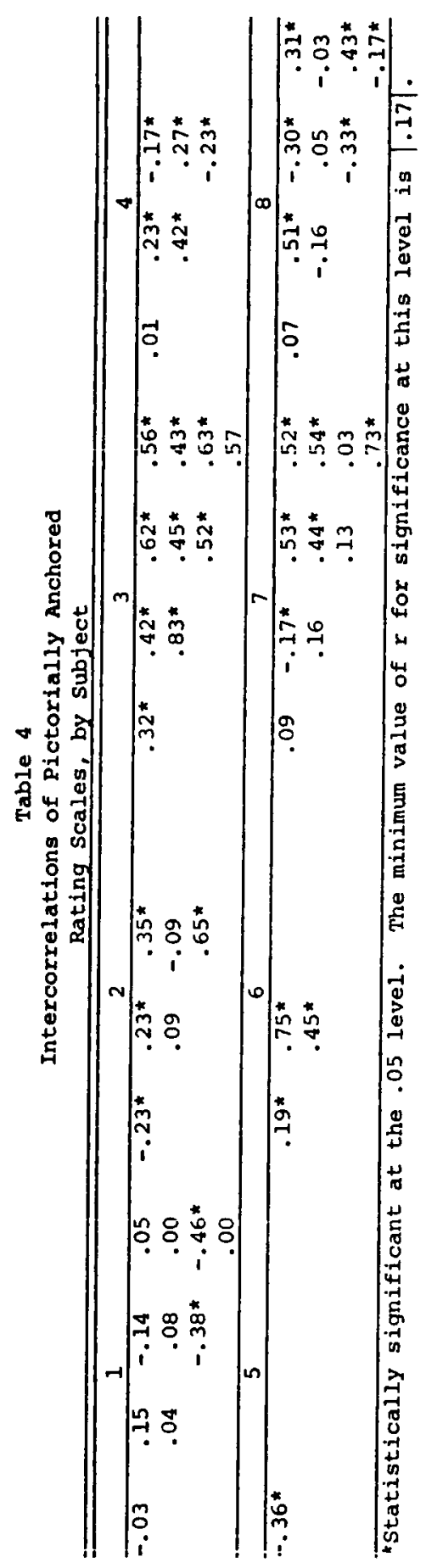

quired to determine why a subject uses one pictorially anchored scale more reliably than another.

\section{Intercorrelations}

Even though the MDS-generated dimensions on which the pictorially anchored scales were based were orthogonal, the measures of the component dimensions in slides of paintings obtained with the pictorially anchored scales were generally not statistically independent. The lack of orthogonality does not seem to be related to difficulty as measured by its subjective rating nor by the time required to use the scales; it may be due to the dimensional relationships in the slides that were rated, rather than to deficiencies in the rating scales.

\section{Verbally Anchored Scales as a Context for Evaluating Pictorially Anchored Scales}

Although the results offer some encouragement, it is possible that the usual verbally anchored scales might outperform their pictorially anchored counterparts. Therefore, it would be useful to compare the characteristics of pictorially anchored scales designed to measure the same perceptual dimensions. The investigator must attempt to insure that both types of scales are measuring the same dimensions; if not, comparison of anchor types will be clouded by potential differences in the dimensions per se.

One approach for obtaining appropriate verbal anchors would be to have subjects and/or experts supply verbal descriptions of the pictures that the MDS analysis located at farthest opposites from the center of the perceptual space. These descriptions would provide the raw material from which verbal anchors could be developed. A different approach would be to use the Rep Test (Kelly, 1955), which is based on perceived similarities and dissimilarities, to generate verbally anchored scales. Ratings on these scales could then be used to select pictures for use as anchors. 


\section{Standard Instead of Tailor-Made Scales}

In this study, pictorially anchored scales were tailor-made for each subject. They were developed as part of an effort to understand the perceptual and judgmental processes of eight specific individuals, and the intent was to generalize only to the population of stimuli with respect to one individual at a time, not to a population of people. However, many investigators may wish to generalize their results to identifiable groups of people. For this purpose, a standard set of pictorially anchored scales should be developed for use by a large number of subjects. There would seem to be two major approaches for accomplishing this: One is to use the average responses of many individuals as input to some dimension-uncovering routine; another is to process judgments of many individuals at once through an individual differences procedures such as INDSCAL. Because of the apparent idiosyncratic nature of visual aesthetic perception, an approach that does not assume similar perceptual structures across subjects would seem more desirable. Such an approach, and one that is almost certain to produce more scales than the first two, would be to combine into one set the nonredundant scales produced by many individual dimension-uncovering analyses. An investigation comparing the characteristics of tailor-made and standard scales would be useful in evaluating the worth of the extra effort involved in developing and using the tailor-made scales.

\section{Other Stimuli}

The rationale for using pictorially anchored scales is the shared nonverbal, visually perceived nature of their anchors and of the stimuli rated through their use. It would be useful to extend the methodology beyond nonrepresentational paintings to other types of nonverbal, visually perceived stimuli, such as those noted at the beginning of this article (e.g., human faces, manner of dress, and styled products such as automobiles).

\section{Predictive Validity}

Since a subject's global reactions to a painting or to other stimulus (e.g., how long he/she looks at it) should be, at least in part, a function of the stimulus' perceived components, it would be desirable to use the measures obtained with the pictorially anchored scales to predict such reactions. Further work should evaluate the predictive validities of the measures obtained with pictorially anchored scales for various types of stimuli. Of particular interest would be the degree to which intercorrelations such as those observed restrict the predictive validities of a subject's set of scales when a theoretically related global reaction is the criterion variable.

\section{References}

Alexander, C. A result in visual aesthetics. British Journal of Psychology, 1960, 51, 357-371.

Arnheim, R. Toward a psychology of art. Berkeley: University of California Press, 1966.

Ashmore, J. Some differences between abstract and nonobjective painting. Journal of Aesthetics and Art Criticism, 1955, 13, 486-495.

Bannister, D., \& Mair, J. M. M. The evaluation of personal constructs. New York: Academic Press, Inc., 1968.

Berleant, A. The sensuous and the sensual in aesthetics.Journal of Aesthetics and Art Criticism, 1964, 23, 185-192.

Berlyne, D. E. Aesthetics and psychobiology. New York: Appleton-Century-Crofts, 1971.

Berlyne, D. E. Studies in the new experimental aesthetics. New York: Wiley, 1974.

Carroll, J. D., \& Chang, J. J. Analysis of individual differences in multidimensional scaling via $n$-way generalization of Eckart-Young decomposition. Psychometrika, 1970, 35, 283-319.

Chandler, A. R. Beauty and human nature. New York: D. Appleton-Century Co., Inc., 1934.

Child, I. L. Esthetics. In G. Lindzey \& E. Aronson (Eds.), The handbook of social psychology (2nd ed.). Reading, MA: Addison-Wesley, 1969.

Einhorn, H. J., \& Koelb, C. A psychometric study of literary-critical judgment. Unpublished paper, University of Chicago, 1974.

Fechner, G. T. Ueber die Fragen des goldenen Schnitts. Archiv. f.d. zeichneden Kuenste (Naumann-Weigel), 1865, 11, 100-112. 
Gibson, J. J. The senses considered as perceptual systems. Boston: Houghton Mifflin Co., 1966.

Henze, D. F. The work of art. The Journal of Philosophy, 1957, 54, 429-442.

Kelly, G. A. Psychology of personal constructs. New York: Norton, 1955.

Kruskal, J. B. Multidimensional scaling by optimizing goodness of fit to a nonmetric hypothesis. Psychometrika, 1964, 29, 15-21. (a)

Kruskal, J. B. Nonmetric multidimensional scaling: A numerical method. Psychometrika, 1964, 29, 115-129. (b)

Lindauer, M. S. Preference for abstract art as a function of complexity and free and controlled exposure time. Proceedings of the annual convention of the American Psychological Association, 1971, 6, 409-410.

Mirels, H. L., \& Efland, A. D. A cognitive approach to the assessment of esthetic responses (Final Report, U.S. Office of Education Project No. 90047). Columbus: Ohio State University, 1970.

Moles, A. Information theory and esthetic perception (Joel E. Cohen, trans.) Urbana: The University of Illinois Press, 1966.

Munro, T. Form and style in the arts: An introduction to aesthetic morphology. Cleveland: The Press of Case Western Reserve University, 1970.

Nidorf, L. J., \& Argabrite, A. H. Aesthetic com- munication: I. Mediating organismic variables. Journal of General Psychology, 1970, 82, 179-193.

Nunnally, J. C. Psychometric theory. New York: McGraw-Hill, 1967.

Nunnally, J. C., Lemond, L. C., \& Wilson, W. H. Studies of voluntary visual attention-theory, methods, and psychometric issues. Applied Psychological Measurement, 1977, 1, 203-218.

Ornstein, R. E. The psychology of consciousness. New York: Penguin, 1975.

Wright, B., \& Rainwater, L. The meaning of color. Journal of General Psychology, 1962, 67, 89-99.

Zenzen, M. J. Ground for aesthetic exper ience. Journal of Aesthetics and Art Criticism, 1976, 34, 469-477.

\section{Acknowledgments}

The author thanks Eugene Alesch of the department of Art at Texas Tech University for his help in selecting and providing the stimuli for this research.

\section{Author's Address}

Arthur D. Beard, Department of Marketing and Hospitality Services Administration, Central Michigan University, Mt. Pleasant, MI 48859. 